\title{
Produtividade de grãos de cafeeiro conilon de diferentes grupos de maturação pelo procedimento REML/BLUP
}

\author{
Grain yield of coffee conilon different maturity groups by \\ REML / BLUP
}

\author{
Cíntia Machado de Oliveira Moulin Carias ${ }^{*}$; Marcelo Antonio Tomaz ${ }^{2}$; \\ Maria Amélia Gava Ferrão ${ }^{3}$; Aymbiré Francisco Almeida da Fonseca ${ }^{3}$; \\ Romário Gava Ferrão ${ }^{4}$; Leandro Simões Azevedo Gonçalves ${ }^{5}$
}

\section{Resumo}

Este trabalho objetivou estimar parâmetros genéticos e avaliar a produtividade de grãos de clones de café Conilon de três grupos de maturação pelo procedimento REML/BLUP. Foram estudados 20 clones de café Conilon de maturação precoce, 20 de maturação intermediária e 20 de maturação tardia para a característica produtividade de grãos. Para cada grupo, representados por épocas distintas de maturação dos frutos foi conduzido um experimento instalado em blocos ao acaso, com quatro repetições, parcelas com cinco plantas, no espaçamento de $3,0 \mathrm{~m} \mathrm{X} \mathrm{1,2} \mathrm{m} \mathrm{com} 2.777$ plantas/ha ${ }^{-1} \mathrm{e}$ as avaliações, realizadas nos anos de 2006, 2007, 2008 e 2009, por ocasião da colheita, conduzidos e cedidos pelo Instituto Capixaba de Pesquisa, Assistência Técnica e Extensão Rural (Incaper). A herdabilidade média $(0,28$; $0,38)$ e o valor da acurácia $(0,68 ; 0,69)$ da seleção de clones foram superiores para o grupo de maturação precoce e intermediário, respectivamente. No grupo tardio, a precisão experimental não foi satisfatória e conseqüentemente, uma baixa acurácia na inferência sobre as médias genotípicas, refletindo na dificuldade de seleção. Os clones 14 e 17 do grupo precoce e 20,14 e 17 do grupo intermediário ocuparam as primeiras colocações na média da característica produtividade e, também foram alocadas nas mesmas posições para MHVG (Estabilidade de Valores Genéticos), PRVG (Adaptabilidade de Valores Genéticos) e MHPRVG (Estabilidade e Adaptabilidade de Valores Genéticos), indicando uma alta produtividade, estabilidade e adaptabilidade frente às variações nas quatro safras para esses genótipos. A interação clones x safras foi significativo para os três grupos de maturação, caracterizando uma situação de interação do tipo complexa, que é problemática para o melhorista devido à inconsistência da superioridade dos clones frente a variações das safras. Os resultados conjuntos evidenciaram superioridade do grupo intermediário em relação ao precoce e tardio.

Palavras-chave: Coffea canephora, clones, produtividade, REML/BLUP, adaptabilidade e estabilidade de produção

1 Discente de Doutorado do Programa de Pós-graduação em Genética e Melhoramento de Plantas, Universidade Estadual Norte Fluminense Darcy Ribeiro, UENF, Campos dos Goytacazes, RJ. E-mail: ciintiia@yahoo.com.br

2 Prof. da Universidade Federal do Espírito Santo, UFES, Alegre, ES. E-mail: tomaz@cca.ufes.br

3 Pesquisadores da Empresa Brasileira de Pesquisa Agropecuária, EMBRAPA CAFÉ/INCAPER, Brasília, DF. E-mail: maria. ferrao@embrapa.br; aymbire.fonseca@embrapa.br

4 Pesquisador do Instituto Capixaba de Pesquisa, Assistência Técnica e Extensão Rural, INCAPER, Vitória, ES. E-mail: romario@ incaper.es.gov.br

5 Prof. do Dept ${ }^{\mathrm{o}}$ de Agronomia, Universidade Estadual de Londrina, Centro de Ciências Agrárias, UEL, Londrina, PR. E-mail: leandrosag@uel.br

* Autor para correspondência 


\begin{abstract}
This study aimed to estimate genetic parameters and evaluate the grain yield clones coffee Conilon three maturity groups by REML / BLUP. We studied 20 clones of coffee Conilon early ripening, maturation 20 intermediate and 20 late maturing for the characteristic grain yield. For each group, represented by different times of ripening of fruits installed an experiment was conducted in a randomized block design with four replications, plots with five plants spaced $3.0 \mathrm{~m} \mathrm{X} 1.2 \mathrm{~m}$ with 2777 plantas/ha-1e evaluations were made in the years 2006, 2007, 2008 and 2009, during harvest, conducted and ceded by the Institute Capixaba Research, Technical Assistance and Rural Extension (Incaper). The average heritability $(0.28$, $0.38)$ and the value of accuracy $(0.68,0.69)$ of clone selection were higher for the group of early and intermediate maturity, respectively. In the late group, the experimental accuracy was not satisfactory and therefore, a low accuracy in inference about the mean genotypic, reflecting the difficulty of selection. Clones 14 and 17 of the early group and 20,14 and 17 of the intermediate group occupied the first places in the middle of the feature and productivity were also allocated in the same positions for MHVG (Genetic Stability of Values), PRVG (Adaptability of Genetic Values) and MHPRVG (Stability and Adaptability of Genetic Values), indicating a high yield, stability and adaptability to variations in four crops for these genotypes. The interaction clones x crops was significant for the three maturity groups, characterizing a complex type of interaction, which is problematic for the breeder due to inconsistency of the superiority of clones against variations of crops. The combined results showed superiority of the group over the intermediate early and late.
\end{abstract}

Key words: Coffea canephora, clones, productivity, REML/BLUP, adaptability and yield stability

\section{Introdução}

Aatividade café apresentaimportante papel sócioeconômico para o Brasil, que é o maior produtor e exportador da cultura no mundo e o segundo maior consumidor (RESENDE et al., 2001; FERREIRA et al., 2005; CECON et al., 2008). A produção brasileira estimada para 2012 é de 52,27 milhões de sacas de 60 quilos do produto beneficiado, sendo que cerca de $70 \%$ do café negociado no mundo é Coffea arabica L. (café arábica) e 30\% Coffea canephora Pierre ex A. Froehner. (café Conilon) (CONAB, 2012).

No Brasil, o Estado do Espírito Santo destacase como o maior produtor de café Conilon, que corresponde a aproximadamente $78 \%$ da produção nacional, seguido pelos Estados de Rondônia e Bahia (CONAB, 2012). As características, alto potencial produtivo, grandes rusticidade e maior quantidade de sólidos solúveis totais, em comparação o café arábica, o que faz da espécie ser desejada pelos melhoristas (FERREIRA et al., 2005).

Devido à relevância desta espécie, desde 1985 a EMCAPA (Empresa Capixaba de Pesquisa
Agropecuária), hoje INCAPER (Instituto Capixaba de Pesquisa, Assistência Técnica e Extensão Rural), iniciou o programa de melhoramento genético de C. canephora, var. Conilon, contribuindo para o aumento da produtividade e melhoria da qualidade da produção. Dentre os resultados, destaca-se a obtenção e o lançamento das variedades EMCAPA 8111 (ciclo precoce); EMCAPA 8121 (ciclo intermediário) e EMCAPA 8131 (ciclo tardio) (BRAGANÇA et al., 1993; BRAGANÇA et al., 2001), seguidas das variedades EMCAPA 8141Robustão Capixaba (FERRÃO et al., 2000a), EMCAPER 8151- Robusta Tropical (FERRÃO et al., 2000b) e Vitória Incaper 8142 (FONSECA et al., 2004).

A C. canephora sendo uma espécie vegetal perene, possuem aspectos biológicos típicos que tornam o seu melhoramento genético distinto de culturas anuais, como: longos ciclos produtivo e reprodutivo, efeito da bienalidade de produção (que é caracterizado pela variação dos anos com alta e baixa produção), necessidade de maiores áreas experimentais, longevidade em produtividade. Assim, por ser um experimento longo no campo, 
o melhorista precisa ficar atentos quanto à redução da sobrevivência das plantas, fato que, associado à sobreposição de gerações, tende a gerar dados desbalanceados para uso na estimação de parâmetros genéticos e na predição de valores genéticos individuais.

A confiabilidade dos resultados demanda rigor e precisão nos métodos de seleção; uso de avaliações repetidas em cada indivíduo ao longo do tempo e uso de métodos especiais de estimação de parâmetros genéticos e de predição de valores genéticos.

Atualmente, o procedimento analítico padrão recomendado para os estudos em genética quantitativa e também para a prática da seleção em plantas perenes é o REML/BLUP, ou seja, a estimação de componentes da variância por máxima verossimilhança restrita (REML) e a predição de valores genéticos pela melhor predição linear nãoviesado (BLUP).

A qual vem sendo aplicada ao melhoramento de espécies florestais como o eucalipto, o pinus, a acácia-negra e a seringueira (RESENDE; HIGA; LAVORANTI, 1993; RESENDE et al., 1996; RESENDE; FERNANDES, 1999; RESENDE et al., 1998; COSTA et al., 1999; KALIL; RESENDE; KALIL, 2000), erva-mate, o cacau e o café (RESENDE et al., 2001; RESENDE; DIAS, 2000; RESENDE, 2001), entre outros. Permitindo levar à interpretação satisfatória de fenômenos biológicos, proporcionando, assim, informações fidedignas e importantes para a obtenção de maiores ganhos genéticos da espécie em estudo.

Este trabalho objetivou estimar parâmetros genéticos e avaliar a produtividade de grãos de clones de café Conilon de diferentes grupos de maturação pelo procedimento REML/BLUP (máxima verossimilhança restrita / melhor predição linear não viesada). A fim de verificar as suas potencialidades para fins de melhoramento genético buscando a obtenção de melhores clones dentro de seus respectivos grupos para dar seguimento ao estudo de melhoramento genético.

\section{Material e Métodos}

Os experimentos foram conduzidos no Incaper na Fazenda Experimental de Bananal do Norte distrito de Pacotuba, município de Cachoeiro de Itapemirim, região sul do Estado do Espírito Santo. O clima da região é classificado como Cwa, com verão chuvoso e inverno seco de acordo com a classificação de Köpen, apresentando alta sazonalidade climática com as estações de baixas e altas pluviosidades bem definidas. A região apresenta precipitação pluvial anual de $1.200 \mathrm{~mm}$, temperatura média anual de $23^{\circ} \mathrm{C}$ e topografia ondulado-acidentada. A altitude é de $140 \mathrm{~m}$, latitude e longitude de $20^{\circ} 45^{\prime} \mathrm{S}$; $41^{\circ} 17^{\prime} \mathrm{W}$ e solo classificado como Latossolo Vermelho Amarelo distrófico.

Foram realizados três experimentos de diferentes ciclos de maturação de Coffea canephora Pierre ex A. Froehner. (café Conilon): 20 genótipos de ciclo de maturação precoce, 20 de ciclo intermediário e 20 de ciclo de maturação tardio. Os ensaios foram instalados em maio de 2004, sendo conduzidos pelos dois primeiros anos para o completo desenvolvimento vegetativo das plantas.

Utilizou-se para cada ensaio o delineamento de blocos ao acaso, com quatro repetições e cinco plantas/parcela e, no espaçamento de 3,0 $\mathrm{m}$ entre linhas e $1,2 \mathrm{~m}$ entre plantas, perfazendo uma população de 2.777 plantas por hectare. As avaliações foram realizadas nos anos de 2006, 2007, 2008 e 2009, por ocasião da colheita, totalizando quatro colheitas, o que representa um número adequado para predição do comportamento de genótipos de Conilon. A caraterística considerada neste trabalho foi à produtividade de grãos (sacas de 60 quilogramas por hectare).

Os componentes de variância e os parâmetros genéticos e fenotípicos e valores genéticos foram obtidos através do programa genético estatístico SELEGEN - REML/BLUP, usando o modelo 55, onde à avaliação em um só local e em várias safras (ambiente), associado ao um modelo misto de interação genótipo x safra (RESENDE, 2002). 
O modelo estatístico para a avaliação de clones no delineamento de blocos ao acaso, com uma observação por parcela e em várias safras foi:

$$
y=x b+Z g+w c+e,
$$

em que $y, b, g, c, e=$ vetores de dados de efeitos fixos ( blocos), efeitos genotípicos de clones (aleatório), efeitos da interação genótipo x safra (aleatório) e de erros aleatórios, respectivamente; e $\mathrm{x}, \mathrm{Z}$ e $\mathrm{w}=$ matrizes de incidência para $\mathrm{b}, \mathrm{g}$ e c, respectivamente.

O número total de clones avaliados nos três experimentos foi 60 . Assim, os efeitos de genótipos foram considerados como aleatórios, conforme Resende e Duarte (2007), que recomendam tratar efeitos genotípicos como aleatórios quando o número de tratamentos for igual ou superior a 10.

Distribuições e estruturas de médias e variâncias:

$$
E\left(\begin{array}{l}
y \\
g \\
c \\
e
\end{array}\right)=\left[\begin{array}{l}
X b \\
0 \\
0 \\
0
\end{array}\right] ; \quad \operatorname{Var}\left[\begin{array}{l}
g \\
c \\
e
\end{array}\right]=\left[\begin{array}{ccc}
I \sigma_{g}^{2} & 0 & 0 \\
0 & I \sigma_{c}^{2} & 0 \\
0 & 0 & I \sigma_{e}^{2}
\end{array}\right]
$$

As equações do modelo misto para estimar os efeitos fixos e predizer os efeitos aleatórios são dadas por:

$\left[\begin{array}{ccc}X^{\prime} X & X^{\prime} Z & X^{\prime} W \\ Z^{\prime} X & Z^{\prime} Z+I \lambda_{1} & Z^{\prime} W \\ W^{\prime} X & W^{\prime} Z & W^{\prime} W+I \lambda_{2}\end{array}\right]\left[\begin{array}{l}\hat{b} \\ \hat{g} \\ \hat{c}\end{array}\right]=\left[\begin{array}{c}X^{`} y \\ Z^{`} y \\ W^{\prime} y\end{array}\right]$ onde:

$$
\begin{aligned}
& I_{1}=\frac{\sigma_{e}^{2}}{\sigma_{g}^{2}}=\frac{1-h_{g}^{2}-c^{2}}{h_{g}^{2}} ; \\
& I_{2}=\frac{\sigma_{e}^{2}}{\sigma_{c}^{2}}=\frac{1-h_{g}^{2}-c^{2}}{c^{2}}
\end{aligned}
$$

em que: $h_{g}^{2}=\frac{\sigma_{g}^{2}}{\sigma_{g}^{2}+\sigma_{c}^{2}+\sigma_{e}^{2}}=$ herdabilidade individual, no sentido amplo no bloco;

$c^{2}=\frac{\sigma_{c}^{2}}{\sigma_{g}^{2}+\sigma_{c}^{2}+\sigma_{e}^{2}}=$ coeficiente de determinação dos efeitos da interação genótipo x safras;

$\sigma_{g}^{2}=$ variância genotípica entre clones;

$\sigma_{c}^{2}=$ variância da interação genótipo x safra;

$\sigma_{e}^{2}=$ variância residual entre parcelas;

$r g=\frac{\sigma_{c}^{2}}{\sigma_{g}^{2}+\sigma_{c}^{2}}=\frac{h_{g}^{2}}{h_{g}^{2}+h_{c}^{2}}=$ correlação genotípica dos materiais genéticos, através das safras.

Estimadores de componentes de variância por REML via algaritmo EM:

$$
\begin{aligned}
& \hat{\sigma}_{e}^{2}=\left[y^{\prime} y-\hat{b}^{\prime} X^{\prime} y-\hat{g}^{\prime} Z^{\prime} y-\hat{c}^{\prime} W{ }^{\prime} y\right] /[N-r(x)] \\
& \hat{\sigma}_{g}^{2}=\left[\hat{g}^{\prime} \hat{g}^{\prime}+\hat{\sigma}_{e}^{2} t r C_{2}\right] / q
\end{aligned}
$$

$\widehat{\sigma}_{c}^{2}=\left[\widehat{c}^{\prime} c+\hat{\sigma}_{e}^{2} \operatorname{tr} C_{3}\right] / s$, em que:

$C_{22}$ e $C_{33}$ advém de:

$C^{-1}\left[\begin{array}{lll}C_{11} & C_{12} & C_{13} \\ C_{21} & C_{22} & C_{23} \\ C_{31} & C_{32} & C_{33}\end{array}\right]^{-1}=\left[\begin{array}{lll}C_{11} & C_{12} & C_{13} \\ C_{21} & C_{22} & C_{23} \\ C_{31} & C_{32} & C_{33}\end{array}\right]$

$\mathrm{C}=$ matriz dos coeficientes das equações de modelo misto;

$\operatorname{tr}=$ operador traço matricial;

$\mathrm{r}(\mathrm{x})=$ posto da matricial $\mathrm{X}$;

$\mathrm{N}, \mathrm{q}, \mathrm{s}=$ número total de dados, número de clones e número de combinações genótipo $x$ safra, respectivamente. 
Nesse modelo, os valores genotípicos preditos livres da interação, considerando-se todas as safras, são dados por $\mathrm{u}+\mathrm{g}$, onde $\mathrm{u}$ representa a média de todas as safras. Para cada safra j, os valores genotípicos são preditos por $u j+g+$ ge, onde uj é a média da safra $\mathrm{j}$.

A seleção conjunta por produtividade, estabilidade e adaptabilidade dos clones baseouse na estatística denominada Média Harmônica da Performance Relativa dos Valores Genéticos (MHPRVG) preditos, conforme descrito por Resende (2004). A MHPRVG conduz a resultados semelhantes aos obtidos pelos métodos descritos por Lin e Binns (1988) e Annicchiarico (1992), contudo, no contexto genotípico e não no contexto fenotípico.

Esse modelo 55 parcimonioso misto univariado, de efeitos principais (G) e interação (GxE), reproduz, aproximadamente, via $\mathrm{G}+\mathrm{Ge}$, os resultados do modelo Blup multivariado, conforme Resende (2004). É importante relatar que o Blup dos efeitos ge eliminam os chamados ruídos da interação genótipo $\mathrm{x}$ ambiente. Isto pode ser visto, considerando-se a predição Blup obtida a partir de uma tabela de dupla entrada, com genótipos (g) e safra (e) contendo as médias de cada genótipo, em cada ambiente. O modelo associado a esta tabela é o seguinte:

$\left(y_{i j}\right)=\mu+g_{i}+e_{j}+g e_{i j}+\varepsilon_{i j}$, em que é o resíduo associado às médias em cada ambiente.

$h_{g}^{2}=\frac{\sigma_{g}^{2}+\sigma_{g e}^{2} / E}{\sigma_{g}^{2}+\sigma_{g e}^{2} / E+\sigma_{e}^{2} / E}$, herdabilidade dos efeitos de genótipos.

$h_{g e}^{2}=\frac{\sigma_{g e}^{2}}{\sigma_{g e}^{2}+\sigma_{e}^{2}}$, herdabilidade dos efeitos da interação g x e. $h_{e}^{2}=\frac{\sigma_{e}^{2}+\sigma_{g e}^{2} / E}{\sigma_{e}^{2}+\sigma_{g e}^{2} / G+\sigma_{e}^{2} / G}, \quad$ coeficiente de

determinação dos efeitos de safras.

$\mathrm{G}$ e E referem-se aos números de safras e de genótipos, respectivamente, e é a variância residual associada às médias Yi.

Verifica-se, por estes índices, que o BLUP de ge considera a herdabilidade dos efeitos da interação $g$ x e, ou seja, elimina os ruídos ou efeitos residuais, por ocasião do processo de predição de ge.

\section{Resultados e Discussão}

Pela estimativa dos parâmetros genéticos observa-se baixa magnitude dos valores genéticos entre os clones dos diferentes grupos de maturação, tendo o maior valor da herdabilidade média dos clones $\left(\hat{h}_{m c}^{2}\right)$ para o grupo de maturação intermediário, seguido pelo precoce e tardio, com 0,38, 0,28 e 0,032, respectivamente (Tabela 1). Segundo Fehr (1987) a herdabilidade elevada está associada com maior variância genética aditiva, menor variação ambiental e menor interação genótipo e ambiente, no entanto, o grupo tardio teve resultados discrepantes aos demais, o que mostra uma marcante influência ambiental. O valor da acurácia da seleção de clones (Acclon) foi de 0,68, 0,69 e 0,18 para os grupos precoce, intermediário e tardio, respectivamente, atestando a adequação da seleção para os clones dos grupos de maturação precoce e intermediária, enquanto para o grupo tardio essa precisão não foi satisfatória refletindo na dificuldade de seleção, podendo estar associados a causas como longevidade do ciclo, grande áreas experimentos, respostas distintas dos genótipos ao estresse hídrico e altas temperaturas, e respostas diferenciadas dos materiais a ventos e podas (FERRÃO et al., 2008) (Tabela 1). Esse parâmetro tem como propósito de corroborar no correto ordenamento das cultivares para fins de seleção e na eficácia da inferência acerca do valor genotípico da cultivar, devido a não 
depender apenas da magnitude da variação residual e do número de repetições, mas também da proporção entre as variações de natureza genética e residual associadas ao caráter em avaliação (RESENDE, 2002; RESENDE; DUARTE, 2007).

A variância residual entre parcelas $\left(\hat{\sigma}_{\theta}^{2}\right)$ foi de 199,73, 209,17 e 252,68 para os grupos de maturação precoce, intermediário e tardio, respectivamente; no qual, implicou erros equivalentes de 28,58, 21,86 e $33,54 \%$, respectivamente a variabilidade fenotípica total. São resíduos de natureza aleatória (não sistemática), que ocorrem na experimentação agrícola e podem comprometer os dados tomados para avaliações (Tabela 1).

Tabela 1. Estimativas de parâmetros genéticos para a produtividade de grãos oriunda da avaliação de três grupos (20 genótipos de ciclo de maturação precoce, 20 de ciclo intermediário e 20 de ciclo de maturação tardio), em quatro safras, no município de Cachoeiro do Itapemirim, ES - Incaper.

\begin{tabular}{cccc}
\hline Parâmetros genéticos $^{1 /}$ & \multicolumn{3}{c}{ Grupos de maturação } \\
\cline { 2 - 4 }$\hat{\sigma}_{g}^{2}$ & Precoce & Intermediário & Tardio \\
$\hat{\sigma}_{\text {parc }}^{2}$ & 44,35 & 97,44 & 4,28 \\
$\hat{\sigma}_{\text {int }}^{2}$ & 4,13 & 25,42 & 3,88 \\
$\hat{\sigma}_{\theta}^{2}$ & 450,55 & 624,76 & 492,55 \\
$\hat{\sigma}_{f}^{2}$ & 199,73 & 209,17 & 252,68 \\
$\hat{h}_{a}^{2}$ & 698,76 & 956,79 & 753,41 \\
$\mathrm{c}^{2}$ parc & $0,0634 \pm 0,0398$ & $0,1018 \pm 0,0505$ & $0,0057 \pm 0,0119$ \\
$\mathrm{c}^{2}$ int & 0,005 & 0,0265 & 0,0051 \\
$\hat{h}_{m c}^{2}$ & 0,64 & 0,66 & 0,66 \\
Acclon & 0,28 & 0,38 & 0,032 \\
$\operatorname{rg}($ safras $)$ & 0,68 & 0,69 & 0,18 \\
Média geral (u) & 0,09 & 0,13 & 0,008 \\
\hline
\end{tabular}

${ }^{1 /} \hat{\sigma}_{g}^{2}=$ variância genética entre clones; $\hat{\sigma}_{\text {parc }}^{2}=$ variância ambiental entre parcelas; $\hat{\sigma}_{\text {int }}^{2}=$ variância da interação clones x safras; $\hat{\sigma}_{\theta}^{2}=$ variância residual entre parcelas;

$\widehat{\sigma}_{f}^{2}=$ variância fenotípica individual; $\widehat{h}_{a}^{2}=$ herdabilidade individual no sentido restrito do bloco; $\mathrm{c}^{2}$ parc $=$ coeficiente de determinação dos efeitos de parcela; $\mathrm{c}^{2}$ int $=$ coeficiente de determinação dos efeitos da interação clones $\mathrm{x}$ safras;

$\widehat{h}_{m p}^{2}=$ herdabilidade da média dos clones; Acclon = acurácia da seleção de clones, assumindo estande completo; e rg(safras) $=$ correlação genética através das safras.

Fonte: Elaboração dos autores.

A interação clones $x$ safras foi de grande e tardio, respectivamente) e baixas correlações magnitude para os três grupos de maturação, genotípicas através das safras $(0,09,0,13$ e 0,008 conforme revelado pelos altos coeficientes de para os grupos precoce, intermediário e tardio, determinação da interação clones x safras $(0,64$, respectivamente) (Tabela1). Isto caracteriza 0,66 e 0,66 para os grupos precoce, intermediário uma situação de interação do tipo complexa, a 
qual é problemática para o melhorista devido à inconsistência da superioridade dos clones frente a variações das safras. Essa alta interação é devido ao efeito da bienalidade, que apesar desse fenômeno ser mais pronunciado no café arabica, mas também ocorre no café conilon, Para minimizar os efeitos da interação clones x safras e ter maior previsibilidade do desempenho são necessários à identificação de clones que tenham uma maior capacidade de recuperação de uma safra para outra.

A média geral (u) dos genótipos em estudo apresenta produtividade $65,67,62,56$ e 62 sacas/ $\mathrm{ha}^{-1}$, para os grupos precoce, intermediário e tardio (Tabelas 1). Estes resultados evidenciam a alta capacidade produtiva do café Conilon, confirmandose, assim, os resultados obtidos por Rodrigues et al. (2012). Fonseca et al. (2004), citam que não se pode atribuir essa alta produtividade apenas ao material genético estudado, mas também a um conjunto de outras tecnologias que foram geradas pela pesquisa e adequadas ao cafeicultor, como poda, densidade de plantas por área, adubação, irrigação, tratos culturais e fitossanitários, entre outras.

$\mathrm{Na}$ seleção dos clones com melhores valores genotípicos, livres da interação $(\mathrm{u}+\mathrm{g})$, para média de todas as safras, obteve-se uma classificação, em função dos componentes das médias. Entre estes, os cinco primeiros clones foram 14, 17, 10, 15 e 9 do grupo precoce, enquanto para o grupo intermediário foram os clones 20, 14, 17, 9 e 12 (Tabelas 2 e 3). Os ganhos genéticos obtidos com a seleção dos referidos clones foram, respectivamente: 12,70, $9,94,8,16,7,00$ e $6,22 \%$ para o grupo precoce; e $19,06,17,32,14,74,13,31$ e $12,27 \%$ para o grupo intermediário.

Tabela 2. Estimativas de valores genotípicos para a característica produtividade de grãos oriunda da avaliação de 20 clones de café Conilon do grupo de maturação precoce em quatro safras, no município de Cachoeiro do Itapemirim, ES - Incaper.

\begin{tabular}{|c|c|c|c|c|c|}
\hline \multirow{2}{*}{ Clones } & \multicolumn{4}{|c|}{ Anos Agrícolas } & \multirow{2}{*}{ Médias } \\
\hline & 2006 & 2007 & 2008 & 2009 & \\
\hline 1 & 61,07 & 59,28 & 86,63 & 79,32 & 71,57 \\
\hline 2 & 46,78 & 76,23 & 40,80 & 63,37 & 56,80 \\
\hline 3 & 38,12 & 74,29 & 25,06 & 60,51 & 49,49 \\
\hline 4 & 42,28 & 67,22 & 51,91 & 40,52 & 50,48 \\
\hline 5 & 75,92 & 54,30 & 42,61 & 16,46 & 47,32 \\
\hline 6 & 50,30 & 38,20 & 80,39 & 67,18 & 59,02 \\
\hline 7 & 39,42 & 56,91 & 44,05 & 97,75 & 59,53 \\
\hline 8 & 38,31 & 69,94 & 67,88 & 113,46 & 72,40 \\
\hline 9 & 58,91 & 64,43 & 72,88 & 94,78 & 72,75 \\
\hline 10 & 39,59 & 64,58 & 88,63 & 112,35 & 76,29 \\
\hline 11 & 44,59 & 53,08 & 72,40 & 50,96 & 55,26 \\
\hline 12 & 63,39 & 73,33 & 70,01 & 60,83 & 66,89 \\
\hline 13 & 55,53 & 78,57 & 65,39 & 63,03 & 65,63 \\
\hline 14 & 60,38 & 86,97 & 74,69 & 158,82 & 95,21 \\
\hline 15 & 39,54 & 62,30 & 61,01 & 133,00 & 73,96 \\
\hline 16 & 48,01 & 38,23 & 56,92 & 98,63 & 60,45 \\
\hline 17 & 49,09 & 74,03 & 77,60 & 128,85 & 82,39 \\
\hline 18 & 52,16 & 83,73 & 75,86 & 72,26 & 71,00 \\
\hline 19 & 60,56 & 74,62 & 83,07 & 66,37 & 71,15 \\
\hline 20 & 49,19 & 44,39 & 68,14 & 61,53 & 55,81 \\
\hline
\end{tabular}

Fonte: Elaboração dos autores. 
Tabela 3. Estimativas de valores genotípicos para a característica produtividade de grãos oriunda da avaliação de 20 clones de café Conilon do grupo de maturação intermediária em quatro safras, no município de Cachoeiro do Itapemirim, ES - Incaper.

\begin{tabular}{|c|c|c|c|c|c|}
\hline \multirow{2}{*}{ Clones } & \multicolumn{4}{|c|}{ Anos Agrícolas } & \multirow{2}{*}{ Médias } \\
\hline & 2006 & 2007 & 2008 & 2009 & \\
\hline 1 & 50,25 & 50,20 & 46,69 & 75,13 & 55,57 \\
\hline 2 & 59,41 & 88,31 & 52,44 & 79,65 & 69,95 \\
\hline 3 & 39,16 & 83,66 & 22,30 & 68,86 & 53,49 \\
\hline 4 & 56,50 & 82,47 & 46,65 & 49,52 & 58,78 \\
\hline 5 & 46,83 & 66,16 & 56,10 & 122,25 & 72,84 \\
\hline 6 & 62,40 & 65,75 & 49,41 & 85,67 & 65,81 \\
\hline 7 & 53,69 & 74,25 & 47,90 & 65,03 & 60,22 \\
\hline 8 & 42,60 & 65,69 & 16,49 & 76,57 & 50,34 \\
\hline 9 & 40,98 & 80,22 & 23,13 & 168,29 & 78,16 \\
\hline 10 & 52,69 & 78,55 & 19,91 & 13,39 & 41,13 \\
\hline 11 & 48,26 & 85,12 & 49,83 & 50,69 & 58,48 \\
\hline 12 & 50,84 & 109,99 & 33,24 & 109,17 & 75,81 \\
\hline 13 & 37,85 & 54,95 & 17,74 & 9,21 & 29,94 \\
\hline 14 & 69,19 & 66,05 & 67,85 & 148,58 & 87,92 \\
\hline 15 & 68,97 & 80,80 & 56,37 & 24,36 & 57,63 \\
\hline 16 & 59,19 & 99,79 & 10,83 & 46,39 & 54,05 \\
\hline 17 & 56,23 & 95,10 & 70,57 & 87,10 & 77,25 \\
\hline 18 & 53,55 & 73,43 & 29,90 & 52,34 & 52,30 \\
\hline 19 & 49,66 & 87,68 & 55,15 & 39,48 & 57,99 \\
\hline 20 & 60,10 & 83,37 & 88,12 & 142,88 & 93,62 \\
\hline
\end{tabular}

Fonte: Elaboração dos autores.

Em relação aos valores genotípicos $(\mathrm{u}+\mathrm{g}+$ gem) para a média dos anos, aproveitando o efeito médio da interação, gerou resultados parcialmente discordantes aos métodos em que se capitaliza a estabilidade (MHVG), adaptabilidade (PRVG) e adaptabilidade e estabilidade (MHPRVG) para os clones de café (Tabela 4). Contudo, pode-se verificar que os clones 14 e 17 do grupo precoce e 20, 14 e 17 do grupo intermediário que ocuparam as primeiras colocações, também foram alocadas nas mesmas posições para MHVG, PRVG e MHPRVG, indicando uma alta produtividade, estabilidade e adaptabilidade frente às variações nas safras para esses clones. 
Tabela 4. Estabilidade de valores genotípicos (MHVG), adaptabilidade de valores genotípicos (PRVG) e estabilidade e adaptabilidade de valores genotípicos (MHPRVG) para a característica produtividade de grãos proveniente da avaliação de 20 clones de café Conilon dos grupos de maturação precoce e 20 clones de café Conilon dos grupos intermediário, em quatro safras, no município de Cachoeiro do Itapemirim, ES - Incaper.

\begin{tabular}{|c|c|c|c|c|c|c|c|}
\hline \multicolumn{4}{|c|}{ Grupo de Clones de Maturação Precoce } & \multicolumn{4}{|c|}{ Grupo de Clone de Maturação Intermediário } \\
\hline Clones & MHVG & PRVG & MHPRVG & Clones & MHVG & PRVG & MHPRVG \\
\hline 1 & 69,69 & 72,49 & 70,82 & 1 & 53,66 & 57,33 & 55,03 \\
\hline 2 & 53,49 & 57,44 & 54,43 & 2 & 66,91 & 70,65 & 70,46 \\
\hline 3 & 41,61 & 49,61 & 42,63 & 3 & 41,30 & 50,55 & 47,04 \\
\hline 4 & 48,50 & 51,92 & 48,26 & 4 & 55,98 & 60,30 & 57,65 \\
\hline 5 & 34,54 & 52,39 & 31,37 & 5 & 64,03 & 72,65 & 67,56 \\
\hline 6 & 54,51 & 59,65 & 55,48 & 6 & 63,35 & 67,19 & 65,89 \\
\hline 7 & 52,72 & 57,86 & 55,38 & 7 & 58,52 & 61,49 & 60,94 \\
\hline 8 & 62,55 & 69,94 & 66,80 & 8 & 35,58 & 47,47 & 41,45 \\
\hline 9 & 70,47 & 72,74 & 72,45 & 9 & 46,49 & 71,25 & 54,63 \\
\hline 10 & 65,65 & 73,99 & 70,08 & 10 & 25,54 & 41,21 & 25,48 \\
\hline 11 & 53,55 & 56,32 & 53,98 & 11 & 55,35 & 59,79 & 57,17 \\
\hline 12 & 66,52 & 68,92 & 66,29 & 12 & 58,82 & 71,55 & 66,85 \\
\hline 13 & 64,63 & 66,98 & 65,09 & 13 & 19,09 & 30,47 & 18,57 \\
\hline 14 & 83,78 & 92,20 & 88,33 & 14 & 78,34 & 88,95 & 80,77 \\
\hline 15 & 61,30 & 70,58 & 65,55 & 15 & 46,70 & 61,99 & 44,60 \\
\hline 16 & 53,56 & 59,32 & 55,58 & 16 & 28,42 & 50,88 & 34,35 \\
\hline 17 & 73,36 & 79,99 & 77,62 & 17 & 74,14 & 79,19 & 77,08 \\
\hline 18 & 68,81 & 71,68 & 70,24 & 18 & 47,15 & 52,12 & 50,61 \\
\hline 19 & 70,15 & 72,73 & 70,51 & 19 & 53,33 & 60,33 & 53,70 \\
\hline 20 & 54,21 & 56,65 & 54,96 & 20 & 85,14 & 95,90 & 88,07 \\
\hline
\end{tabular}

Fonte: Elaboração dos autores.

\section{Conclusões}

1. A interação clones $\mathrm{x}$ safras foi de grande magnitude para os três grupos de maturação, caracterizando uma situação de interação do tipo complexa, que é problemática para o melhorista devido à inconsistência da superioridade dos clones frente a variações das safras.

2. Os resultados conjuntos evidenciaram superioridade do grupo intermediário em relação ao precoce e tardio.

3. Na seleção dos clones com melhores valores genotípicos, os de numero 14 e 17 do grupo precoce e 20,14 e 17 do grupo intermediário ocuparam as primeiras colocações, indicando uma alta produtividade, estabilidade e adaptabilidade frente às variações de ano para ano nas quatro safras estudadas.

\section{Agradecimentos}

Ao INCAPER E EMBRAPA/CAFÉ por ter permitido à utilização dos dados experimentais $\mathrm{e}$ pela parceria estabelecida.

\section{Referências}

ANNICCHIARICO, P. Cultivar adaptation and recomendation from alfafa trials in northern Italy. Journal of Genetics and Breeding, Roma, v. 46, n. 1, p. 269-278, 1992.

BRAGANÇA, S. M.; CARVALHO, C. H. S. de; FONSECA, A. F. A.; FERRÃO, R. G. 'EMCAPA 8111', 'EMCAPA 8121', 'EMCAPA 8131': variedades clonais de café conilon para o Estado do Espírito Santo. Pesquisa Agropecuária Brasileira, Brasília, v. 36, n. 5, p. 765-770, 2001. 
BRAGANÇA, S. M.; CARVALHO, C. H. S. de; FONSECA, A. F. A.; FERRÃO, R. G.; SILVEIRA, J. S. M. 'EMCAPA 8111', 'EMCAPA 8121', 'EMCAPA 8131': primeiras variedades clonais de café conilon lançadas para o Estado do Espírito Santo. Vitória: Emcapa, 1993. 2 p. (Emcapa. Comunicado técnico, 68).

CECON, P. R.; SILVA, F. F. E.; FERREIRA, A.; FERRÃO, R. G.; CARNEIRO, A. P. S.; DETMANN, E.; FARIA, P. N.; MORAIS, T. S. S. Análise de medidas repetidas na avaliação de clones de café 'Conilon'. Pesquisa Agropecuária Brasileira, Brasília, v. 43, n. 9, p. 1171-1176, 2008.

COSTA, R. B.; RESENDE, M. D. V. de; ARAÚJO, A. J.; GONÇALVES, P. S. BLUP individual e índice multiefeitos aplicados ao melhoramento da seringueira. In: SIMPÓSIO SOBRE RECURSOS GENÉTICOS PARA A AMÉRICA LATINA E CARIBE, 2., 1999, Brasília. Anais... Brasília: Embrapa Recursos Genéticos e Biotecnologia, 1999. 1 CD-ROM.

COMPANHIA NACIONAL DE ABASTECIMENTO CONAB. Acompanhamento da safra brasileira de café: safra 2012, segunda estimativa, maio/2012. Brasília: CONAB, 2012. 18 p.

FEHR, W. R. Principles of cultivars development. New York: Macmillan, 1987. 536 p.

FERRÃO, R. G.; FONSECA, A. F. A.; FERRÃO, M. A. G.; BRAGANÇA, S. M. 'EMCAPER 8151'- robusta tropical: primeira variedade melhorada de café conilon de propagação por sementes para o Estado do Espírito Santo. Vitória, ES: Emcaper, 2000b. 2 p. (Emcaper. Documento, 103).

FERRÃO, R. G.; FONSECA, A. F. A.; SILVEIRA, J. S. M.; FERRÃO, M. A. G.; BRAGANÇA, S. M. 'EMCAPA 8141'- Robustão Capixaba, variedade clonal de café conilon tolerante à seca, desenvolvida para o estado do Espírito Santo. Revista Ceres, Viçosa, MG, v. 47, n. 273, p. 555-559, 2000a.

FERRÃO, R. G.; CRUZ, C. D.; FERREIRA, A.; CECON, P. R.; FERRÃO, M. A. G.; FONSECA, A. F. A.; CARNEIRO, P. C. S.; SILVA, M. F. Parâmetros genéticos em café Conilon. Pesquisa Agropecuária Brasileira, Brasília, v. 43, n. 1, p. 61-69, jan. 2008.

FERREIRA, A.; CECON, P. R.; CRUZ, C. D.; FERRÃO, R. G.; SILVA, M. F. da; FONSECA, A. F. A.; FERRÃO, M.A.G. Seleção simultânea de Coffea canephora por meio da combinação de análise de fatores e índices de seleção. Pesquisa Agropecuária Brasileira, Brasília, v. 40, n. 12, p. 1189-1195, 2005.
FONSECA, A. F. A.; FERRÃO, M. A. G.; FERRÃO, R. G.; VERDIN FILHO, A. C.; VOLPI, P. S.; ZUCATELI, F. Conilon Vitória - Incaper 8142: variedade clonal de café Conilon. Vitória, ES: Incaper, 2004. 24 p. (Incaper. Documento, 127).

KALIL, A. N.; RESENDE, M. D. V.; KALIL, G. P. C. Componentes de variância e predição de valores genéticos em seringueira pela metodologia de modelos mistos (REML/BLUP). Pesquisa Agropecuária Brasileira, Brasília, v. 35, n. 9, p. 1883-1887, 2000.

LIN, C. S.; BINNS, M. R. A superiority measure of cultivar performance for cultivar x location data. Canadian Journal of Plant Science, Ottawa, v. 68, n. 3, p. 193-198, 1988.

RESENDE, M. D. V. de. Genética biométrica e estatística no melhoramento de plantas perenes. Brasília: Embrapa Informação Tecnológica; Colombo: Embrapa Floresta, 2002. $975 \mathrm{p}$.

Melhoramento de espécies perenes. In: NASS, L. L. VALOIS, A. C. C.; MELO, I. S. de; VALADARESINGLIS, M. C. (Ed.). Recursos genéticos e melhoramento de plantas. Rondonópolis, MT: Fundação MT, 2001. $1183 \mathrm{p}$.

Métodos ótimos na análise de experimentos de campo. Colombo: Embrapa Floresta, 2004. 100 p.

RESENDE, M. D. V. de; DIAS, L. A. S. Aplicação da metodologia de modelos mistos (REML/BLUP) na estimação de parâmetros genéticos e predição de valores genéticos aditivos e genotípicos em espécies frutíferas. Revista Brasileira de Fruticultura, Jaboticabal, v. 22, n. 1, p. 44-52, 2000.

RESENDE, M. D. V. de; DUARTE, J. B. Precisão e controle de qualidade em experimentos de avaliação de cultivares. Pesquisa Agropecuária Tropical, Goiânia, v. 37, n. 3, p. 182-194, 2007.

RESENDE, M. D. V. de; FERNANDES, J. S. C. Procedimento BLUP individual para delineamentos experimentais aplicados ao melhoramento florestal. Revista de Matemática e Estatística, São Paulo, v. 17, n. 1, p. 89-107, 1999.

RESENDE, M. D. V. de; FURLANI JÚNIOR, E.; MORAES, M. L. T.; FAZUOLI, L. C. Estimação de parâmetros genéticos e predição de valores genotípicos no melhoramento do cafeeiro pelo procedimento REML/ BLUP. Bragantia, Campinas, v. 60, n. 3, p. 185-194, 2001. 
RESENDE, M. D. V. de; HIGA, A. R.; LAVORANTI, O. J. Predição de valores genéticos no melhoramento de eucalyptus - melhor predição linear. In: CONGRESSO FLORESTAL BRASILEIRO, 7., 1993, Curitiba. Anais...

São Paulo: Sociedade Brasileira de Silvicultura, v. 1, 1993. p. 144-147.

RESENDE, M. D. V. de; MORA, A. L.; HIGA, A. R.; PALUDZYSZYN FILHO, E. Efeito do tamanho amostral na estimativa da herdabilidade em espécies perenes. Floresta, Curitiba, v. 28, n. 1-2, p. 51-63, 1998.
RESENDE, M. D. V. de; PRATES, D. F.; JESUS, A.; YAMADA, C. K. Estimação de componentes de variância e predição de valores genéticos pelo método da máxima verossimilhança restrita (REML) e melhor predição linear não viciada (BLUP) em Pinus. Boletim de Pesquisa Florestal, Colombo, v. 32-33, n. 3233, p.1845, 1996.

RODRIGUES, W. N.; TOMAZ, M. A.; FERRÃO, R. G.; FERRÃO, M. A. G.; FONSECA, A. F. A.; MIRANDA, F. D. Estimativa de parâmetros genéticos de grupos de clones de café. Coffee Science, Lavras, v. 7, n. 2, p. 177186, 2012. 
